\title{
Are We Monitoring Urine Output for the Surgical Patients in Ward Settings While Indicated or Planned?
}

\author{
Ramprasad Rajebhosale, Mohammad Miah, Daniel Centea, Cindy Cleto, Rabia Yusuf, \\ Prabhu Ravi, Rajesh Paul, Najam Husain, Pradeep Thomas
}

Queens Hospital Burton, Burton on Trent, UK

Email:mohammad.miah1@nhs.net

How to cite this paper: Rajebhosale, R. Miah, M., Centea, D., Cleto, C., Yusuf, R., Ravi, P., Paul, R., Husain, N. and Thomas, P. (2020) Are We Monitoring Urine Output for the Surgical Patients in Ward Settings While Indicated or Planned?. Open Access Library Journal, 7: e6498.

https://doi.org/10.4236/oalib.1106498

Received: June 3, 2020

Accepted: July 17, 2020

Published: July 20, 2020

Copyright $\odot 2020$ by author(s) and Open Access Library Inc.

This work is licensed under the Creative Commons Attribution International License (CC BY 4.0).

http://creativecommons.org/licenses/by/4.0/

\section{(c) (i) Open Access}

\begin{abstract}
Introduction: Early recognition of acute kidney injury (AKI), better understanding of its pathogenesis, and development of preventing strategies appear to be potential areas of improvement of patient's prognosis. The decrease of glomerular filtration rate and urine output in response to a decrease of renal blood flow is classically referred as pre-renal azotaemia, which can evolve into structural damage if renal hypoperfusion persists. In this line, urine output often is used as a marker of AKI but also to guide fluid resuscitation in critically ill patients. Methods: Our audit objective is to find out the number of patients undergoing major surgery or advised by the surgeon, do they have urine output monitoring in place. Prospective data were collected over 2 weeks by patients' record review and bedside examination. Results: A total of 77 patients were included and among them 53 patients had urine output accurately monitored and 24 patients were not monitored for urine output. Conclusion: As UO monitoring can be early indicator of illness, deterioration or impending adverse event like AKI and not doing so would lead to major complications of electrolyte imbalance putting patient safety at risk. From our study it is evident that all patients did not have their urine output monitored.
\end{abstract}

\section{Subject Areas \\ Clinical Trials}

\section{Keywords}

Urine Output, Surgical Patients, UO Monitoring 


\section{Introduction}

Acute renal failure or acute kidney injury (AKI) is defined by an acute decline of glomerular filtration rate (GFR). Occurrence of AKI is associated with substantial in-hospital mortality, exceeding $50 \%$ when AKI is part of a multiple organ failure syndrome [1] [2]. Therefore, early recognition of AKI, better understanding of its pathogenesis, and development of preventing strategies appear to be potential areas of improvement of patient's prognosis. The decrease of glomerular filtration rate and urine output in response to a decrease of renal blood flow is classically referred as pre-renal azotaemia, which can evolve into structural damage if renal hypoperfusion persists. In this line, urine output often is used as a marker of AKI but also to guide fluid resuscitation in critically ill patients. Changes in urine output are indicators of risk, and it is important that these biomarkers are monitored alongside a "track and trigger" system (NEWS). Recognising and responding to these changes will ensure appropriate and quick intervention to prevent acute kidney injury developing. Adults in hospital at risk of acute kidney injury include those:

- who have non-elective admissions

- who have any major planned interventions, [NICE guideline CG3] for definitions of surgery grades)?

And also, in order to avoid iatrogenic complications of intravenous fluid regimes, all patients on maintenance fluid should ideally have: frequent vital signs

- Daily weight

- Strict input and output charts, including drain and nasogastric losses

- Note: Urine output should be greater than $0.5-1.0 \mathrm{ml} / \mathrm{kg} / \mathrm{hour}$ (depending on age)

- At least daily serum biochemistry and glucose monitoring

- At least daily medical review to determine ongoing need for IV therapy We followed the NICE Guideline [AKI, Quality standard (QS76), Published on 18 December 2019]. This recommended the followings:

- The urine output should be recorded at admission or in the initial assessment and then as part of routine monitoring.

- Frequency of urine output monitoring will also depend on clinical circumstances.

- The decrease of glomerular filtration rate and urine output in response to a decrease of renal blood flow is classically referred as pre-renal azotaemia, which can evolve into structural damage if renal hypoperfusion persists.

- In this line, urine output often is used as a marker of AKI but also to guide fluid resuscitation in critically ill patients.

\section{Materials and Method}

Our audit objective is to find out the number of patients undergoing major surgery or advised by the physician do they have urine output monitoring in place. Prospective data were collected over 2 weeks by patients' record review and bed- 
side examination. Patients who were admitted under General Surgery in both male and female wards from $1^{\text {st }}$ of January 2020 till $16^{\text {th }}$ of January 2020 at Queens Hospital Burton and were planned or indicated for UO monitoring were included. All patients from 18 to 90 years of age, who had emergency surgical admissions and were listed for elective colon cancer surgeries, were incorporated. Those who underwent day case surgery were excluded from the study. Audit was registered with the audit department of the Trust.

\section{Results}

A total of 77 patients were included. Minimum age was 19 years and maximum age was 90 years. 34 were male patients and 43 were female patients. Among them 53 patients had urine output accurately monitored and 24 patients were not monitored for urine output [Figure 1]. 13 patients had documented plan in place for urine output monitoring. Surprisingly, there were additional 40 patients found who had urine output monitored even there was no plan for UO monitoring. Total numbers of catheterized patients were 15 and all of them had their urine output monitored.

\section{Discussion}

Reduced urine output is widespread among critically ill surgical patients as well as postoperative patients and can reflect a reduction in creatinine clearance. A drop in renal blood flow and/or a decline in renal perfusion pressure is a key determining factor of GFR. In hypovolemic patients, such as postoperative patients prompt fluid resuscitation is essential to avoid additional worsening of renal function. The preference of the nature of fluid also appears to be vital, as colloids rise the oncotic pressure and might decrease filtration rate. UO monitoring is related with improved recognition of moderate to severe AKI and decreased prevalence of fluid overload and is independently associated with reduced mortality in surgical patients suffering from AKI. Our results should good practice of monitoring the urine output in critically ill surgical patients.

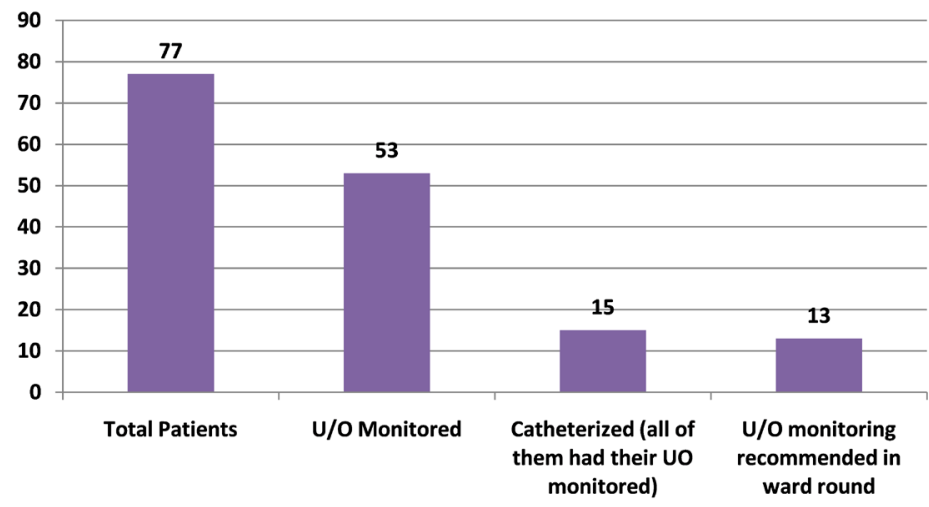

Figure 1. Bar graph showing urine output monitoring during in patient stay. 


\section{Conclusion}

Decreasing urine output is common among critically ill patients and can mirror a decrease in creatinine clearance. In hypovolemic states, prompt fluid resuscitation is needed to prevent further deterioration of renal function. From our study it is evident that all patients did not have their urine output monitored. As UO monitoring can be early indicator of illness, deterioration or impending adverse event like AKI and not doing so would lead to major complications of electrolyte imbalance putting patient safety at risk.

\section{Disclosure and Ethics}

Authors have no conflict of interest to disclose and there was no funding involved in this study. This study was registered with the relevant department of the hospital. No intervention was carried other than the usual protocol and no patient identifiable data were used. Thus, patients verbally consented.

\section{Conflicts of Interest}

The authors declare no conflicts of interest regarding the publication of this paper.

\section{References}

[1] Chen, Y.C., Jenq, C.C., Tian, Y.C., Chang, M.Y., Lin, C.Y., Chang, C.C., Lin, H.C., Fang, J.T., Yang, C.W. and Lin, S.M. (2009) Rifle Classification for Predicting In-Hospital Mortality in Critically Ill Sepsis Patients. Shock, 31, 139-145. https://doi.org/10.1097/SHK.0b013e31817d419e

[2] Morgera, S., Schneider, M. and Neumayer, H.H. (2008) Long-Term Outcomes after Acute Kidney Injury. Critical Care Medicine, 36, S193-S197.

https://doi.org/10.1097/CCM.0b013e318168cae2 